Doug Geisler, Eva K. Grebel, and Dante Minniti, eds.

\title{
Star Formation Properties of 100 Star Formation Complexes in 20 Galaxies
}

\author{
F Sakhibov \\ Institute of Astrophysics of Tajik Academy of Sciences, Bukhoro str. 22 \\ Dushanbe, 734042, Tajikistan \\ M.A. Smirnov \\ Institute of Astronomy of Russian Academy of Sciences, Pyatnicskaya \\ 48, Moscow 109017, Russia
}

\begin{abstract}
The simultaneous multiple constraints of the IMF and SFR history based on the observed $U B V R$ colours, Lyman continuum fluxes and chemical abundances $Z$ resolve the IMF-SFR ambiguity in 100 extragalctic star formation complexes in 20 galaxies (SFCs). The separate study of SFCs with different SFR history shows links between IMF parameters and observed properties of SFCs as well as local and global properties of a host galaxy. The SFR history depends on the star density of SFCs. Low density regions demonstrate instantaneous starburst, while in high density SFCs extended star formation bursts are detected. Constrained IMF slopes are in a good agreement with direct observations of IMFs in OB associations of the Milky Way and LMC.
\end{abstract}

\section{Introduction}

Direct observations of IMFs in clusters and associations of the Milky Way and LMC show that the overall shape of the IMF may not depend on local and global properties of a host galaxy: the IMF derived from star counts is an average over metallicity, density, morphological type a to be remarkably uniform from region to region covering star formation that spans a factor of 200 in density. To verify so important conclusion one has to extend the IMF slope estimations to a larger number of star forming regions in galaxies with different densities, metallicity and morphological types. The problem is that the IMF in more distant galaxies remains inaccessible to star counts. The integrated technique based on simultaneous use of multiple constraints of IMF and SFR history parameters (including a regime of star formation) may provide IMF estimations in an enough large sample of star formation complexes (SFCs) in distant galaxies. This procedure results in large uncertainties for individual star forming complexes, but may be adequate for a study of the systematic properties of SFCs in different environments. The relations between star formation characteristics and local and global properties of a host galaxy may provide useful guidance to the theory of star formation in galaxies. The method is discussed in our previous papers (Sakhibov \& Smirnov 2000). We have adopted a power law IMF $\left(f \propto m^{\alpha}\right.$, in 


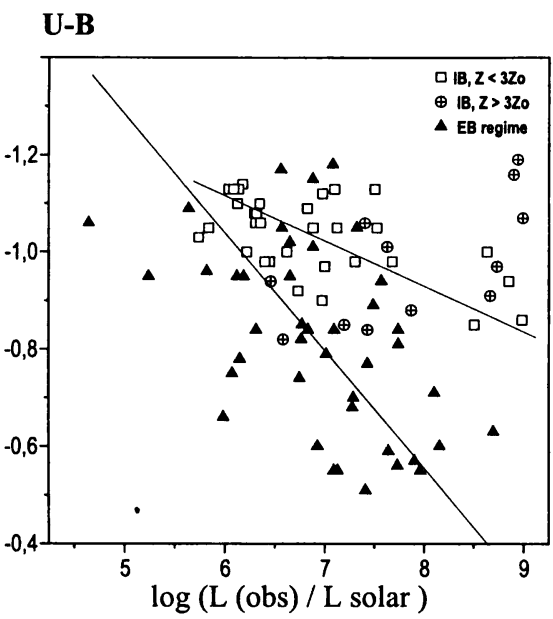

IMF slope $\alpha$

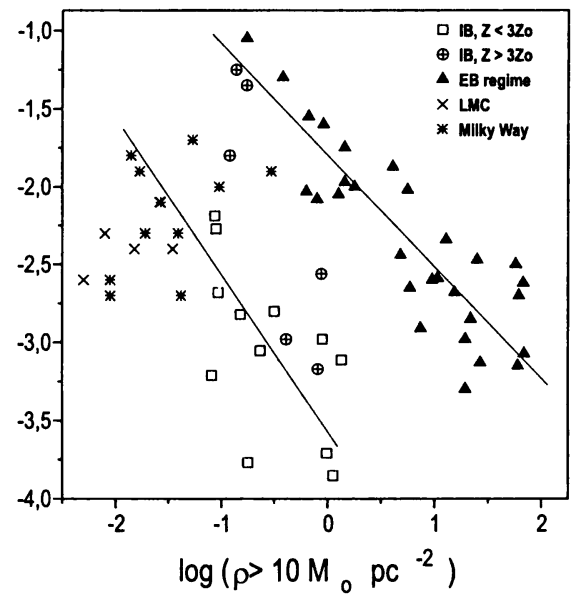

Figure 1. Left: Colour - luminosity diagram of SFCs with different SFR history: IB - squares; EB - triangles. Right: - scatter diagram IMF slope $\alpha$ - surface stellar density of SFCs; the positions of OB associations in the Milky Way (crosses) and LMC (stars) with direct estimated IMF slopes by star counting correspond to positions of IB regions (squares).

our notation $\alpha=-2.35$ for a Salpeter IMF) of intermediate and high mass stars from $M_{\min }=2 M_{\odot}$ to upper mass limits $M_{\max }$; and two limiting models of star formation history: instantaneous burst of age t (IB model) and extended star formation with constant rate and duration $\mathrm{t}$ (EB model) calculated by Piskunov \& Myakutin (1996). The accepted models are not universal and should regarded as useful tools to find links between star formation parameters (IMF, SFR) and observed properties of SFCs (colours, luminosity, sizes) as well as local and global properties of a host galaxy (density, metallicity, morphological type).

\section{The IMF-SFR ambiguity is resolved.}

The problem of the resolution of the IMF-SFR history (regime) ambiguity in an individual star formation complex remains is paramount and should be solved in the first order in case of the integrated technique. In the colour - luminosity diagram (Fig. 1 left) which is model free, we have displayed low correlated $(r \approx 0.5-0.7)$ linear regression equations to show that SFCs with different star formation history demonstrate different average $U-B$ colour indices at fixed luminosity. Starburst (IB) regions (squares) are experienced to have bluer $U-B$ colours at fixed luminosity compare to EB regions (triangles). We do not included in to the statistic analysis the metal-rich IB regions $\left(Z>3 Z_{\odot}\right.$, circles) for the reasons, which are discussed in our main paper (Sakhibov \& Smirnov, 2001). 


\section{IMF and SFR parameters are linked to sizes and colours..}

The $U-B$ colours are sensitive to IMF slope variations in both cases of star formation regime: SFCs with flatter IMF have a bluer $U-B$ colours. $B-V$ colours are not sensitive to the slope $\alpha$ variations. Analysis of the distribution of derived ages $t$ of individual SFCs with their sizes $S$ (pc) pointed out that older SFCs are larger and brighter than younger SFCs in both cases of star formation regime (IB \& EB). Note that the age - size dependence pointed out for star clusters in the LMC by Efremov and Elmegreen (1998) is close to the $t-S$ dependence of extended star formation bursts (EB).

\section{SFR history depends on the star density of SFC.}

Whether there is the density dependence of the IMF is an important question to understanding the star formation processes. In Fig. 1 (right). we plotted IMF slope $\alpha$ as a function of surface stellar densities $\rho\left(>10 M_{\odot} \cdot p c^{-2}\right)$ of studied SFCs. The density range in diagram varies by a factor $10^{4}$, which is 100 times larger than the range of densities in star clusters with direct estimated IMFs by star counting in the Milky Way (stars) and LMC (crosses) (Massey et al. 1995). Fig. 1 (right) shows that position of clusters with direct estimated IMFs (crosses and stars) are in an agreement with the slope - density dependence of IB star forming regions (squares). We excluded from the diagram two associations in the MW (NGC 7235 and Cep OB5) because of low significant slope estimations. The SFR history depends on the star density of SFC. Low density regions demonstrate instantaneous starburst, while in high density SFCs we detected extended star formation bursts. Age and duration of SFR history are higher at high density complexes.

\section{Star formation in SFCs are linked to the morphological type of a host galaxy: older IB regions live in earlier type spirals.}

There is a trend toward old ages of IB regions $\left(Z<3 Z_{\odot}\right)$ in early spirals. Note that the trend in is in the consistent with the trend toward higher luminosities in early type galaxies and the age - luminosity (size) dependence. It seems that the difference of angular momentum between early an late type spirals plays role in the age differences in the case of normal metallicity IB regions.

\section{References}

Efremov Yu. N., Elmegreen. B. 1998, MNRAS, 299, 588.

Massey P., Johnson K.E., DeGoa-Eastwood K. 1995, ApJ, 454, 151.

Piskunov A. E., Myakutin V. I. 1996, AZh 73, 520.

Sakhibov F., Smirnov M.A., 2000, A\&A , 354, 802.

Sakhibov F., Smirnov M.A., 2001, A\&A, in preparation 